\title{
Fibrous dysplasia: rapid malignant transformation into osteogenic sarcoma - A rare occurance
}

\author{
Gon $\mathrm{S}^{1}$, Majumdar $\mathrm{B}^{1}$, Bhattacharyya $\mathrm{A}^{1}$, Bhattacharya $\mathrm{RN}^{2}$ \\ ${ }^{I}$ Department of Pathology, RG Kar Medical College \& Hospital,Kolkata, India \\ ${ }^{2}$ Department of Plastic \& Reconstructive Surgery, RG Kar Medical College \& Hospital, Kolkata, India
}

\section{Keywords:}

Fibrous dysplasia; Malignant transformation;

Osteogenic sarcoma

\begin{abstract}
Malignant transformation of fibrous dysplasia is rare, occurring in less than $1 \%$ of cases with a mean lag period of 13.5 years. We report a case of Osteogenic Sarcoma with chondroid differentiation in a pre-existing Fibrous Dysplasia occurring within one year of surgical resection and without any history of exposure to radiation. To the best of our knowledge and extensive search of literature, malignant transformation of Fibrous Dysplasia in such a short period of time, and without history of radiation exposure has never been reported from India.
\end{abstract}

\section{INTRODUCTION}

Fibrous dysplasia (FD) is a benign bone disorder of an unknown etiology, uncertain pathogenesis and diverse histopathology. ${ }^{1}$ Three major types: monostotic, involving a single bone; polyostotic, having multiple lesions involving multiple bones; and McCune Albright syndrome, a polyostotic form of fibrous dysplasia that also involves endocrine abnormalities have been described. ${ }^{2}$

Though benign, malignant degeneration occurs in less than $1 \%$ of cases of fibrous dysplasia. For unknown reasons, monostotic and craniofacial lesions have the greatest potential for malignant transformation. ${ }^{2}$ We report a case of Osteogenic sarcoma with chondroid differentiation in a pre-existing Fibrous Dysplasia occurring within one year of surgical resection and without any history of exposure to radiation.

\section{Correspondence:}

Dr Sonia Gon, MD

Assistant Professor, Department of Pathology, RG Kar Medical College \& Hospital,Kolkata, India.

Email:drmarur@yahoo.com

\section{CASE REPORT}

A 16-years-old patient presented with a gradually increasing swelling at right side of mandible at outpatient department of Plastic Surgery, R G Kar Medical College \& Hospital, Kolkata. The swelling was non tender, hard in consistency and rapidly growing in nature. Patient had a history of excision of a swelling at same site ten months back which on histopathology was diagnosed as Monostotic FD (fig.1).

Patient's routine haematological and biochemical investigations were within normal limits apart from mildly raised serum alkaline phosphatase levels. There was no evidence of hyperthyroidism, acromegaly, precocious puberty or skin pigmentation. Digital X-Ray of the face showed ground glass opacity in the lower and anterior mandible with irregular calcification, which was consistent with the clinical diagnosis of FD (fig.2). He was subjected to plain \& contrast enhanced CT scan of face which revealed a fibro-osseous lesion measuring $7.7 \times 7.3 \times 7.8 \mathrm{~cm}$ at right side of mandible involving adjacent submental space, right sided submandibular space, parotid space and masticator space. Considerable partially necrotic and heterogeneously enhancing ill-defined soft tissue component of the lesion 


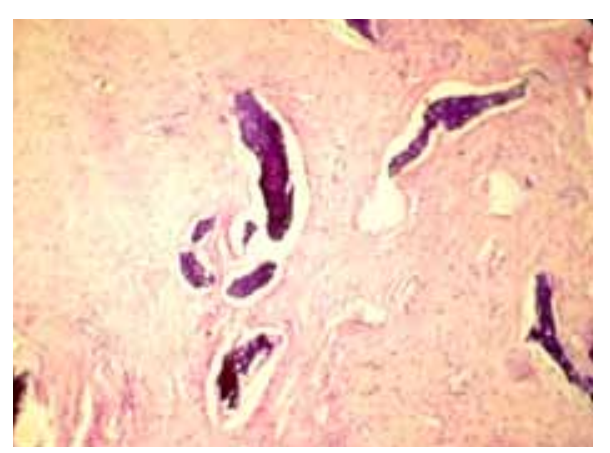

Figure 1: Irregular trabeculae of osteoid along with hypocellular spindle cell stroma (HE stain, X400)

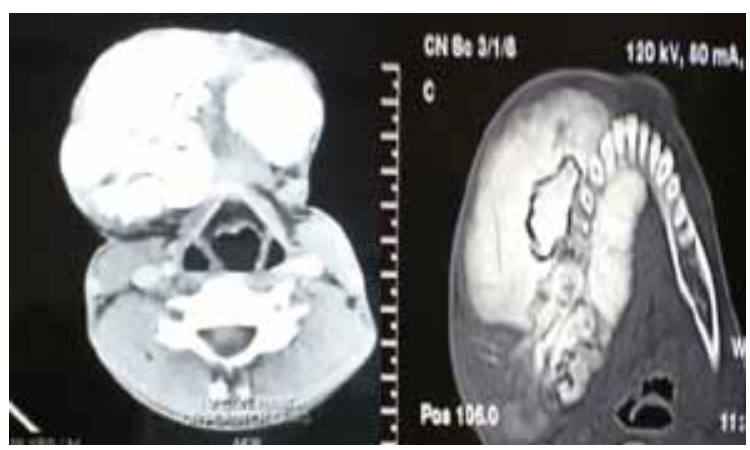

Figure 3: Plain \& contrast enhanced CT scan showing heterogeneously enhancing ill defined fibro-osseous lesion in the mandible involving adjacent spaces and also bone destruction.

with loss of adjacent soft tissue plane and expansion along with destruction of bone was noted, strongly suggesting sarcomatous changes in FD (fig. 3).

Right sided hemi-mandibulectomy with resection of the mass was done and the specimen was sent for histopathological examination.

\section{Histopathological findings}

On gross examination, the specimen consisted of part of mandible with teeth and a bony hard tumour measuring 7 $\mathrm{cm}$ in its greatest diameter. On microscopic examination, the tumour comprised spindle to polygonal cells exhibiting mild to moderate nuclear pleomorphism and osteoid formation. At places, malignant cartilage was also present. Significant mitotic figures were found. Histopathological diagnosis of low grade osteogenic sarcoma of mandible with chondroid differentiation arising from pre-existing FD was made (fig.4).

\section{DISCUSSION}

Malignant transformation of FD of the facial bones and jaw is rarely reported and is usually associated with a history of prior radiation treatment. ${ }^{3}$ Malignant transformation of $\mathrm{FD}$ is rare and involves transformation into osteosarcoma,

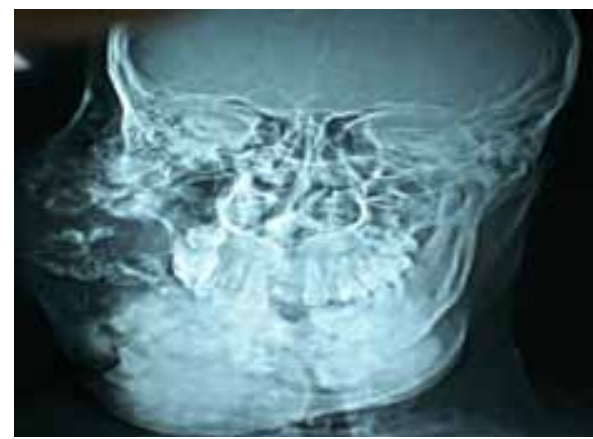

Figure 2: Digital X-ray exhibiting ground glass opacity in the lower and anterior mandible with irregular calcification, consistent with Fibrous Dysplasia.

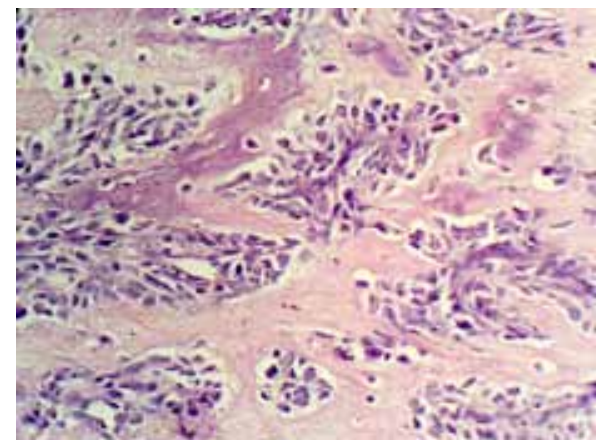

Figure 4: Highly pleomorphic malignant cell with laying down of malignant osteoid. (HE stain, X400) fibrosarcoma or chondrosarcoma, in decreasing order of frequency. ${ }^{4}$ Ruggieri et $\mathrm{al}^{5}$ studied 28 cases of malignant transformation in FD comprising $2.5 \%$ of the total lesions involving bone, out of which $67.8 \%$ were osteogenic sarcoma. Rest $17.8 \%, 10.7 \%$ and $3.6 \%$ were fibrosarcoma, chondrosarcoma and malignant fibrohistiocytoma respectively. Most of the cases (46\%) had a history of prior exposure to radiation. The present case also developed malignant transformation of monostotic FD diagnosed histopathologically as low grade osteogenic sarcoma with chondroid differentiation without any prior exposure to radiation.

Clinically, pain, rapid growth of a lesion and a dramatic elevation of alkaline phosphatase may herald malignant transformation. $^{2}$ The malignant transformation is noted higher among male with polyostotic FD, craniofacial lesions and monostotic FD and also, exposure to radiotherapy increases malignant transformations $>400$ folds. ${ }^{6,7}$ Though the present case, a 16 years old male, did complain of pain and gradually increasing size of the tumour, serum alkaline phosphatase levels were only mildly raised.

In most reported cases, the diagnosis of FD was made in childhood but the malignant tumor developed during the third or fourth decades of life. ${ }^{8}$ The lag between the development of the FD and the sarcoma varied from a 
minimum of 2 years to a maximum of 30 years, the mean being 13.5 years. ${ }^{9}$ Gyu-Tae Kin et a ${ }^{10}{ }^{10}$ recently described a case of malignant transformation of monostotic mandibular FD after two surgical excisions, 20 and 15 years ago, respectively. The present case was only 16 years old and developed malignant transformation in FD within ten months of resection of tumour.

Differentiation of the osteogenic sarcoma, osteomyelitis and FD is difficult on the basis of radiographic features alone. ${ }^{11}$ The most common cross-sectional imaging findings, suggestive of but not pathognomonic for sarcoma development, are the presence of a soft tissue mass and bone destruction. ${ }^{3}$ Histologically, osteosarcomma consist of a malignant undifferentiated stroma and neoplastic osteoid formation along with increased mitotic rate of proliferating stromal cells. The stromal component is characterised by dense cellularity and pleomorphism

The main stay of treatment remains surgery and there is no significant difference in patient survival after curative surgery with radiation treatment and without radiation treatment, with patients being disease free for as long as 5 years and $3 \frac{1}{2}$ years respectively. ${ }^{3}$ Prognosis for malignant change in $\mathrm{FD}$, however, is poor. Most patients died with pulmonary metastasis, and the mean survival period was 3.4 years. $^{8}$

\section{CONCLUSION}

Malignant transformation of FD is well documented in the literature and should be kept as a differential diagnosis when patient complains of recurrent swelling of short duration at the same site. Histopathology is the gold standard to reach at a conclusive diagnosis.

\section{REFERENCES}

1. Ozek C, Gundogan H, Bilkay U, Tokat C, Gurler T, Songur E. Craniomaxillofacial fibrous dysplasia. J Craniofac Surg. 2002;13:382-9.

2. Cholakova R, Kanasirska P, Kanasirski N, Chenchev Iv, Dinkova A. Fibrous dysplasia in the maxillomandibular region - case report. Journal of imab 2010;16:10-3.

3. Kaushik S, Smoker WRK, Frable WJ. Malignant transformation of fibrous dysplasia into chondroblastic osteosarcoma. Skeletal Radiol 2002;31:103-6.

4. Campanacci M, Bertoni F, Capanna R. Malignant degeneration in fibrous dysplasia presentation of 6 cases and review of the literature. Ital J Orthop Traumatol 1979;5:373-81.

5. Ruggieri P, Sim FH, Bond JR, Unni KK. Malignancies in fibrous dysplasia. Cancer 1994;73:1411-24.

6. Ben hadj Hamida F, Jlaiel R, Ben Rayana N et al. Craniofacial fibrous dysplasia: a case report. J Fr Ophtalmol 2005;28:e6

7. Edgerton MT, Persing JA, Jane JA. The surgical treatment of fibrous dysplasia.With emphasis on recent contributions from craniomaxillo-facial surgery. Ann Surg. 1985;202:459-79.

8. Yabut SM, Kenan S, Sissons HA, Lewis MM. Malignant transformation of fibrous dysplasia. A case report and review of the literature. Clin Orthop Relat Res 1998;228:281-9.

9. Schwartz DT, Alpert M. The malignant transformation of fibrous dysplasia. Am J Med Sci 1964;247:1-20.

10. Kim GT, Lee JK, Choi BJ, Kim J, Han SH, Kwon YD. Malignant transformation of monostotic fibrous dysplasia in the mandible. $\mathrm{J}$ Craniofac Surg 2010;21:601-3.

11. Petrikowski CG, Pharoah MJ, Lee L, Grace MG. Radiographic differentiation of osteogenic sarcoma, osteomyelitis, and fibrous dysplasia of the jaws. Oral surg oral med oral pathol oral radiol endod 1995;80:744-50. 